\title{
Optimal treatment for primary benign intratracheal schwannoma: A case report and review of the literature
}

\author{
XIAHUI GE ${ }^{1}$, FENGFENG HAN $^{1}$, WENBIN GUAN ${ }^{2}$, JINYUAN SUN ${ }^{1}$ and XUEJUN GUO ${ }^{1}$ \\ Departments of ${ }^{1}$ Respiratory Medicine and ${ }^{2}$ Pathology, Xinhua Hospital, Shanghai Jiaotong University \\ School of Medicine, Shanghai 200092, P.R. China
}

Received September 4, 2014; Accepted May 27, 2015

DOI: $10.3892 / \mathrm{ol} .2015 .3521$

\begin{abstract}
A 53-year-old male was admitted to Xinhua Hospital (Shanghai, China) due to coughing and dyspnea that had persisted for half a year, with aggravation of chest tightness and dyspnea for 1 week. Chest computed tomography (CT) revealed a mass within the distal third of the trachea. Flexible bronchoscopy confirmed an $\sim 2.0 \mathrm{~cm}$, smooth, tan-colored mass in the trachea, $2 \mathrm{~cm}$ above the carina. Endoscopic resection by argon plasma coagulation combined with electronic snaring was applied, however, recurrence was found 2 weeks later. Finally, the tumor was completely removed by surgery and the post-operative course was uneventful. Since schwannoma is rare in the intrapulmonary region and extremely rare in the trachea, a review of 51 cases of primary tracheal schwannoma previously reported in the English literature was performed. The majority of cases occurred in adults and were usually located in the distal third of the trachea. The predominant tumor size was $1-3 \mathrm{~cm}$ and airway obstruction symptoms were common. Half of the patients were misdiagnosed with asthma, and CT scan and bronchoscopy were contributory to the correct diagnosis. The treatment of choice depended on the patient's condition, however, surgery should be chosen in the event of local recurrence following endoscopic treatment.
\end{abstract}

\section{Introduction}

Primary tracheal tumors are relatively uncommon and 2 out of 3 are malignant or of intermediate malignancy, such as squamous cell carcinoma, adenoid cystic carcinoma and carcinoid tumors, while the remaining third are benign lesions, such as papilloma, hemangioma and hamartoma. Schwannoma and neurofibroma originate from Schwann cells and are usually

Correspondence to: Dr Xuejun Guo, Department of Respiratory Medicine, Xinhua Hospital Affiliated to Shanghai Jiaotong University School of Medicine, 1,665 Kong Jiang Road, Shanghai 200092, P.R. China

E-mail: guoxj1122@hotmail.com

Key words: intratracheal tumor, schwannoma, clinical characteristics, management of schwannoma benign lesions. They rarely occur in the trachea and the exact frequency is unknown. Thus far, limited cases have been reported, of which the majority occur in the lower trachea. Previous reports have indicated that endoscopic and surgical resection have been successfully applied in the treatment of the disease, and as a result, the prognosis of the disease was improved (1-4).

The present study reports the case of a patient with a benign primary tracheal schwannoma who was treated by tracheal resection and reconstruction following recurrence after endoscopic resection. In addition, 51 cases of primary tracheal schwannoma from English literature are reviewed, with emphasis on the clinical characteristics of the disease and the selection of the treatment.

\section{Case report}

A 53-year-old male presented to Xinhua Hospital (Shanghai, China) on August 19 $19^{\text {th }}, 2013$ with coughing and dyspnea that had persisted for half a year, and increasing chest tightness and dyspnea for 1 week. The past medical history was significant for hypertension and diabetes. In the Outpatient Clinic, the patient's spirometry showed a severe obstructive ventilation defect and the following computed tomography (CT) scan of the chest revealed a well-defined soft-tissue mass in the trachea. The patient was admitted to the hospital and a physical examination revealed a blood pressure of $190 / 120 \mathrm{mmHg}$ (normal range, $<130 / 85 \mathrm{mmHg}$ ), a heart rate of 102 beats/min (normal range, 60-100 beats/min), a respiratory rate of 23-38 breaths/min (normal range, $12-20$ breaths/min) and an oxygen saturation of $98 \%$ on room air (normal range, >94\%). Lung auscultation indicated a prolonged expiratory phase and wheezing over the cervical tracheal segment in the inspiratory and expiratory phases. Multiplanar reconstructions and contrast-enhanced CT indicated a mass with minimal enhancement, $\sim 1.5 \mathrm{~cm}$ above the carina and occluding $\sim 90 \%$ of the tracheal lumen, while at the same time smoothly protruding into the esophagus (Fig. 1A and B). Flexible bronchoscopy confirmed the presence of a $2.0-\mathrm{cm}$, round, tan-colored mass in the distal third of the trachea. The mass was covered with several small, discrete vessels on the surface, but was not pulsatile or friable (Fig. 1C). Gastroscopy was applied immediately and there were no abnormalities in the esophagus mucosa. 
A

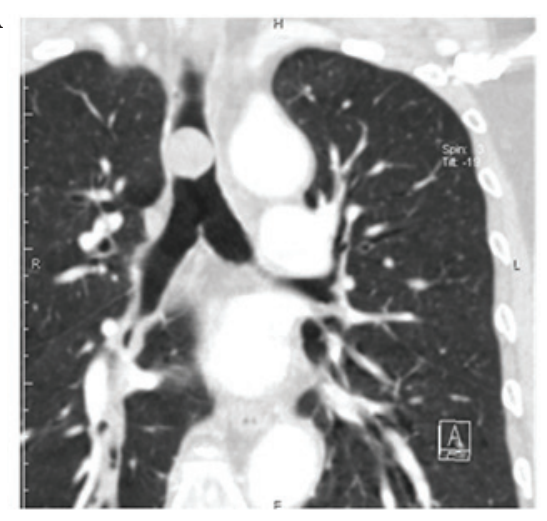

B

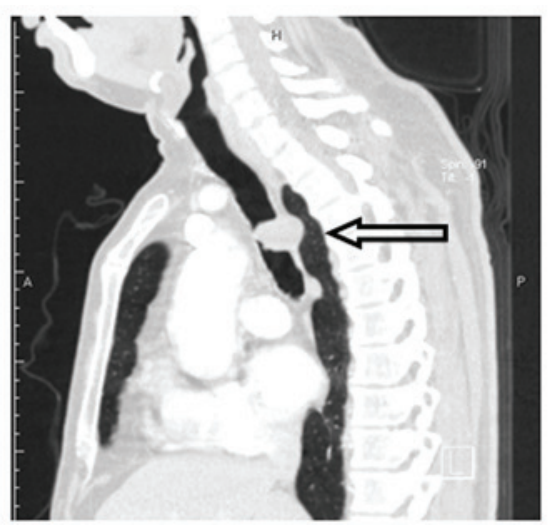

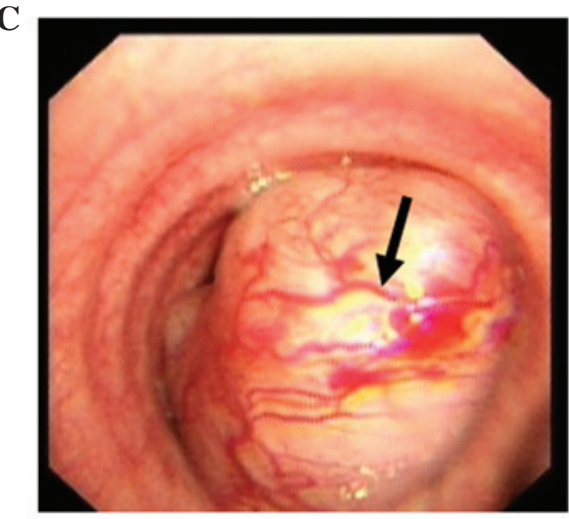

Figure 1. Intratracheal schwannoma on computed tomography scan and bronchoscopy examination. (A) Coronal reconstruction and (B) sagittal reconstruction showing the tumor in the lumen of the trachea (arrow), arising from the posterior tracheal wall. (C) Bronchoscopy revealed that $~ 90 \%$ of the trachea was obstructed by the round, tan-colored mass, which was covered with several small vessels on the surface (arrow).
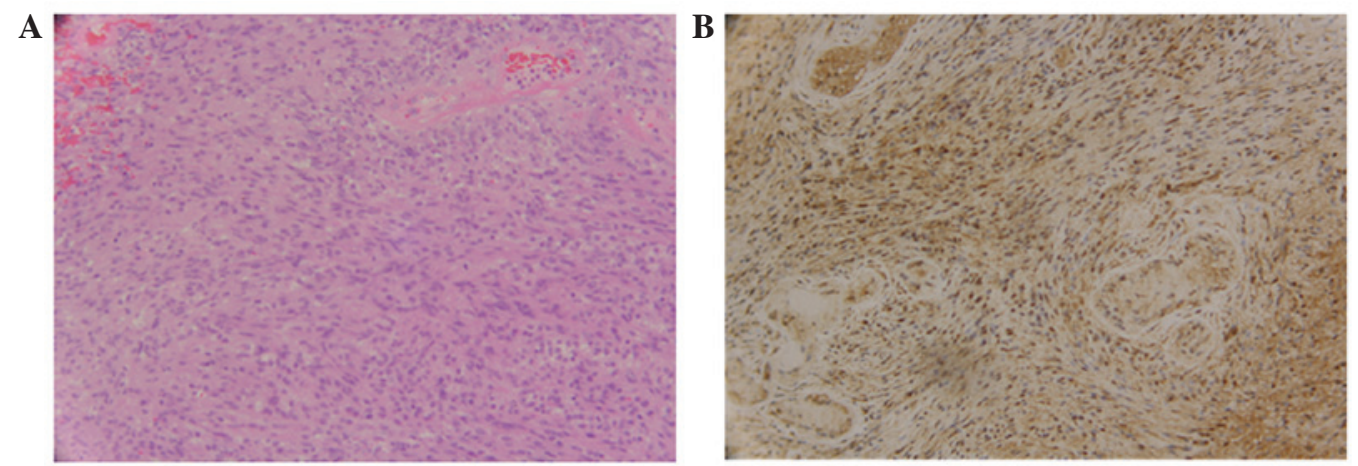

Figure 2. Pathological findings. The tumor was composed of spindled cells and elongated nuclei arranged in a palisading pattern, as shown by (A) hematoxylin and eosin staining (magnification, x100). (B) The tumor showed positivity for S-100 protein on immunohistochemical staining (magnification, x100).

Electrosurgical snaring was used to cut the mass under flexible bronchoscopy. Morphologically, the tumor exhibited an Antoni A growth pattern, composed of spindle cells with elongated palisading nuclei (Fig. 2A). A few hyalinized blood vessels were found, whereas no clear necrosis or mitotic activity was observed within these areas. The tumor demonstrated strong and diffuse nuclear and cytoplasmic positivity for S-100 protein (Fig. 2B) and vimentin, but was negative for cytokeratin, desmin, cluster of differentiation 117 and smooth muscle actin. The histological and immunohistochemical findings were consistent with benign schwannoma. Argon plasma coagulation (APC) combined with electronic snaring was applied twice and $75 \%$ of the mass was removed. The shortness of breath was resolved immediately and the patient was discharged on day 3 post-treatment. However, chest tightness recurred 2 weeks later. Bronchoscopic examination was employed again and a relapse of the mass in the same region of the trachea was confirmed. A $3-\mathrm{cm}$ segment of the trachea was subsequently resected and an anastomosis was performed. The resection margins were free of tumor. There were no post-operative complications and no recurrence in the 1 year follow-up period. At the time of writing, the patient was well with no evidence of disease.

The patient provided written informed consent for the publication of this study. Furthermore, the study was approved by the
Ethics Committee of Xinhua Hospital Affiliated to Shanghai Jiaotong University School of Medicine.

\section{Discussion}

Neurogenic tumors, schwannoma and neurofibroma are the main benign tumors arising from the peripheral nerves. Schwannoma is much less common than neurofibroma, and usually occurs in the head, neck, retroperitoneum, extremities and mediastinum $(5,6)$. The tumor is extremely rare in the trachea, being more frequently reported in the lungs and bronchi.

The present study reviewed the literature between 1950 and 2013, and identified 51 cases of primary tracheal schwannoma (1-3,7-22). In these cases, schwannoma mainly occurred in adults $(78.4 \% ; 40 / 51)$, with a predilection for women (30 females and 20 males; gender of 1 case was not stated). The majority of the cases occurred in the distal third of the trachea, followed by the proximal and then the middle third of the trachea. Of the 51 cases, 26 were reported in Asian populations, followed by North American and European populations. The clinical symptoms and signs depended on the tumor location, size and degree of airway obstruction. Coughing, wheezing and dyspnea as airway obstruction symptoms were 
commonly reported, while hemoptysis, hoarseness and chest pain were also reported in tracheal schwannoma. Of the 51 patients, 15 presented with wheezing or stridor on physical examination. Half of the patients were initially misdiagnosed with asthma, and treatment was delayed for an average of 17 months from the onset of symptoms. The tumor size varied from 1.0 to $4 \mathrm{~cm}$. The tumors were commonly $1-3 \mathrm{~cm}$ and only 3 cases exhibited a tumor of $>3 \mathrm{~cm}$ in diameter in the trachea. In terms of management, 19 patients underwent endoscopic resection of the mass and 29 received surgical resection. Among the patients whose masses were resected by the endoscopic technique, laser with or without a $\mathrm{CO}_{2}$, electronic snaring, APC, cryotherapy, endoscopic excision and microdebridement were applied. However, 4 cases were reported with relapse and were further treated by surgery $(4,23,24)$. In total, $3.9 \%(2 / 51)$ of patients presented with malignant schwannoma. Overall, 3 cases succumbed to complications, including renal insufficiency, post-operative infection and hypoxic brain damage induced by acute airway obstruction.

Pulmonary function and CT were useful for forming an early diagnosis. Pulmonary function, particularly flow-volume curves, showed upper airway obstruction, which prompted the clinicians to proceed with further examinations. In the present case, the severe obstructive ventilation defect on spirometry assisted the locating of the mass in the trachea. CT was used to define the tumor size, location, and intratracheal and extratracheal extension. Bronchoscopy as a further examination technique could directly view the mass in the lumen, and evaluate the extent and vascularity of the lesion and its association to the peritracheal tissue. Bronchoscopy was also useful in the biopsy and incision of the tumor.

As reported previously, tracheal resection and endoscopic excision could be utilized in the management of tracheal schwannoma $(8,21,25)$. In the present patient, the huge sessile mass in the trachea was confirmed by bronchoscopy. In the study by Lee et al (26), a sessile mass in left main bronchus was successfully removed by using APC and rigid bronchoscopy tip, and the patient was only followed up for 4 months. Due to the reluctance of the present patient to accept surgery initially, treatment with endoscopic snaring combined with APC was performed, and an incomplete initial resection was confirmed 2 weeks later. The patient then underwent segmental tracheal resection and tracheal reconstruction, and no recurrence was found in 1 year of follow-up.

The management of tracheal schwannoma should consider the size and extratracheal component of the tumor, the cardiopulmonary function in the patient and the pedicle of the tumor. For patients with pedunculated and completely intraluminal tumors (20), or in those with severe underlying diseases (27), endoscopic resection may provide a reasonable treatment option. For those patients with huge and sessile schwannomas in the trachea and non-cardiopulmonary function restriction, surgical resection is indispensable as an optimal treatment method (3). However, prior reports also indicated that endoscopic resection in schwannoma has the possibility of local recurrence $(4,23,24)$, whereas no relapse was recorded in cases of surgical resection. As for local recurrence following endoscopic resection, surgery should be chosen.

In conclusion, primary tracheal schwannoma is rare. The tumor usually causes airway obstruction and the symptoms of this are common between cases. Pulmonary function, CT and bronchoscopy are useful for the diagnosis. The treatment of choice depends on the condition of the patient, whereas surgery must be chosen when local recurrence follows endoscopic treatment. Although the prognosis of primary tracheal schwannoma is favorable, long-term follow-up after tumor removal is required.

\section{References}

1. Davies MJ, Hall DR and Ross BA: Rare tracheal tumours: Two case reports of primary neurogenic tumours occurring in the trachea. Respir Med 87: 145-146, 1993.

2. Tang LF, Chen ZM and Zou CC: Primary intratracheal neurilemmoma in children: Case report and literature review. Pediatr Pulmonol 40: 550-553, 2005.

3. Righini CA, Lequeux T, Laverierre MH and Reyt E: Primary tracheal schwannoma: One case report and a literature review. Eur Arch Otorhinolaryngol 262: 157-160, 2005.

4. Kasahara K, Fukuoka K, Konishi M, Hamada K, Maeda K, Mikasa K and Kimura H: Two cases of endobronchial neurilemmoma and review of the literature in Japan. Intern Med 42: 1215-1218, 2003.

5. Takeda S, Miyoshi S, Minami M and Matsuda H: Intrathoracic neurogenic tumors-50 years' experience in a Japanese institution. Eur J Cardiothorac Surg 26: 807-812, 2004.

6. Das Gupta TK, Brasfield RD, Strong EW and Hajdu SI: Benign solitary Schwannomas (neurilemomas). Cancer 24: 355-366, 1969.

7. Dutta R, Kumar A, Kaushal S and Choudhary SK: A case of carinal schwannoma resected under cardiopulmonary bypass. Indian J Cancer 48: 366-368, 2011.

8. Melendez J, Cornwell L, Green L and Casal RF: Treatment of large subglottic tracheal schwannoma with microdebrider bronchoscopy. J Thorac Cardiovasc Surg 144: 510-512, 2012.

9. Erol MM, Uzun H, Tekinbas C, Gunduz A, Turedi S and Kosucu P: A case of intratracheal schwannoma presenting at the emergency department with a diagnosis of asthmatic attack. J Emerg Med 39: 589-591, 2010.

10. Shah SS, Karnak D, Shah SN, Biscotti C, Murthy S and Mehta AC: Clinical-pathologic conference in general thoracic surgery: A malignant peripheral nerve sheath tumor of the trachea. J Thorac Cardiovasc Surg 132: 1455-1459, 2006.

11. Pang L C: Primary neurilemoma of the trachea. South Med J 82: 785-787, 1989.

12. Nio M, Sano N, Kotera A, Shimanuki Y, Takeyama J and Ohi R: Primary tracheal schwannoma (neurilemoma) in a 9-year-old girl. J Pediatr Surg 40: E5-E7, 2005.

13. Dincer SI, Demir A, Kara HV, Fener N and Altin S: Primary tracheal schwannoma: A case report. Acta Chir Belg 106: 254-256, 2006.

14. Takeda K, Horiuchi M, Nakaya M, Yamaguchi K and Fujikawa A: Schwannoma of the trachea; a new resection technique. Auris Nasus Larynx 30: 425-427, 2003.

15. Kitagawa H, Kawase H, Wakisaka M, Satou Y, Satou H, Furuta $\mathrm{S}$ and Nakada K: Six cases of children with a benign cervical tumor who required tracheostomy. Pediatr Surg Int 20: 51-54, 2004.

16. Murata T, Shino M, Yasuoka Y and Chikamatsu K: Subglottic Schwannoma: A report of a rare case that was treated with medial thyrotomy. Am J Otolaryngol 34: 569-573, 2013.

17. Hamdan AL, Moukarbel RV, Tawil A, El-Khatib M and Hadi U: Tracheal schwannoma: A misleading entity. Middle East J Anesthesiol 20: 611-613, 2010.

18. Thomas R, Christopher DJ, Thangakunam B and Samuel R: Tracheal schwannoma as a mimic of bronchial asthma. Singapore Med J 53: e95-e96, 2012.

19. Rusch VW and Schmidt RA: Tracheal schwannoma: management by endoscopic laser resection. Thorax 49: 85-86, 1994.

20. Weiner DJ, Weatherly RA, DiPietro MA and Sanders GM: Tracheal schwannoma presenting as status asthmaticus in a sixteen-year-old boy: Airway considerations and removal with the CO2 laser. Pediatr Pulmonol 25: 393-397, 1998.

21. Dorfman J, Jamison BM and Morin JE: Primary tracheal schwannoma. Ann Thorac Surg 69: 280-281, 2000.

22. Nass RL and Cohen NL: Neurilemoma of the trachea. Arch Otolaryngol 105: 220-221, 1979. 
23. Horovitz AG, Khalil KG, Verani RR, Guthrie AM and Cowan DF: Primary intratracheal neurilemoma. J Thorac Cardiovasc Surg 85: 313-317, 1983.

24. Jung YY, Hong ME, Han J, Kim TS, Kim J, Shim YM and Kim H: Bronchial schwannomas: Clinicopathologic analysis of 7 cases. Korean J Pathol 47: 326-331, 2013.

25. Stouffer CW, Allan RW, Shillingford MS and Klodell CT: Endobronchial schwannoma presenting with bronchial obstruction. Interact Cardiovasc Thorac Surg 10: 133-134, 2010.
26. Lee BR, Choi YD, Kim YI, Lim SC and Kwon YS: Endobronchial schwannoma treated by rigid bronchoscopy with argon plasma coagulation. Tuberc Respir Dis 73: 174-177, 2012.

27. Feldhaus RJ, Anene C and Bogard P: A rare endobrochial neurilemmoma (Schwannoma). Chest 95: 461-462, 1989. 